\title{
Solid-liquid phase equilibrium for binary Lennard-Jones mixtures
}

\author{
Monica R. Hitchcock and Carol K. Hall ${ }^{\text {a) }}$ \\ Department of Chemical Engineering, North Carolina State University, Raleigh, \\ North Carolina 27695-7905
}

(Received 8 January 1999; accepted 15 March 1999)

\begin{abstract}
Solid-liquid phase diagrams are calculated for binary mixtures of Lennard-Jones spheres using Monte Carlo simulation and the Gibbs-Duhem integration technique of Kofke. We calculate solidliquid phase diagrams for the model Lennard-Jones mixtures: argon-methane, krypton-methane, and argon-krypton, and compare our simulation results with experimental data and with Cottin and Monson's recent cell theory predictions. The Lennard-Jones model simulation results and the cell theory predictions show qualitative agreement with the experimental phase diagrams. One of the mixtures, argon-krypton, has a different phase diagram than its hard-sphere counterpart, suggesting that attractive interactions are an important consideration in determining solid-liquid phase behavior. We then systematically explore Lennard-Jones parameter space to investigate how solidliquid phase diagrams change as a function of the Lennard-Jones diameter ratio, $\sigma_{11} / \sigma_{22}$, and well-depth ratio, $\epsilon_{11} / \epsilon_{22}$. This culminates in an estimate of the boundaries separating the regions of solid solution, azeotrope, and eutectic solid-liquid phase behavior in the space spanned by $\sigma_{11} / \sigma_{22}$ and $\epsilon_{11} / \epsilon_{22}$ for the case $\sigma_{11} / \sigma_{22}<0.85$. (C) 1999 American Institute of Physics.
\end{abstract}

[S0021-9606(99)51122-7]

\section{INTRODUCTION}

Successful design of melt crystallization processes depends upon knowledge of the equilibrium solid-liquid phase behavior of the mixture to be separated. Although mixture solid-liquid equilibrium can, in principle, be measured at any condition of interest, it would be useful to develop a theory that predicts mixture phase behavior based solely upon knowledge of the components' molecular architecture and intermolecular forces. Such a theory should be able to predict the six types of binary solid-liquid phase diagrams introduced in 1977 by Matsuoka, ${ }^{1}$ a classification based on the analysis of nearly 1500 binary organic systems. The six types of binary solid-liquid phase diagrams are shown in Fig. 1; they include: solid solutions, azeotropes, eutectics with partial solid phase immiscibility, eutectics with complete solid phase immiscibility, peritectics with eutectics, and molecular compounds.

Our intent in this paper is to explore the range of Lennard-Jones parameters that will yield the phase diagrams characteristic of solid solutions, azeotropes, and eutectics with partial solid phase immiscibility. The solid phase in all of these phase diagrams is a substitutionally disordered solid solution, i.e., both species pack with the same crystalline structure and can substitute for one another in any order on the lattice. In future work we will examine the effect of Lennard-Jones parameters on solid-liquid equilibrium for cases in which the solid phase is an ordered crystal and the associated phase diagram contains either a peritectic with eutectic or a molecular compound.

Several workers have developed molecularly-based

\footnotetext{
a) Author to whom correspondence should be addressed: electronic mail: hall@turbo.che.ncsu.edu
}

theories of solid-liquid phase equilibria using simple models, such as the hard-sphere model or the Lennard-Jones 12-6 potential model, in an effort to understand how molecular size differences and interactions influence the phase behavior of a mixture. Cottin and Monson ${ }^{2}$ developed a cell theory for binary solid solutions based on the early cell model of Lennard-Jones and Devonshire for single component solids. In this model, each molecule is assumed to move within the cage formed by its nearest neighbors. A cell is characterized by the identity of its central molecule and by the composition and geometrical arrangement of its nearest neighbors. Assuming that the product of the cell partition functions is equal to the configurational partition function, the solid solution free energy can be determined as a function of composition. The liquid solution free energy can be obtained from an equation of state. Once the free energies of both phases are obtained, the convex envelope construction method is used to locate the solid-liquid phase envelope.

The cell theory has been applied to phase diagram calculations for both hard-sphere and Lennard-Jones binary mixtures. Cottin and Monson calculated phase diagrams for binary hard-sphere liquid mixtures coexisting with substitutionally disordered fcc (Ref. 2) and several ordered ${ }^{3}$ binary hard-sphere solids. Cottin and co-workers ${ }^{4,5}$ showed that the cell theory is capable of predicting five of the six solidliquid phase diagrams displayed in Fig. 1. The only type of phase diagram that the cell theory with the hard-sphere potential model does not predict is that for molecular compound formation. The fact that the hard-sphere model can generate five of the six known types of solid-liquid phase diagrams indicates that packing and molecular size differences, which are the major consideration in a hard-sphere mixture, are the dominant factors in determining the shape of solid-liquid phase diagrams in real systems. 

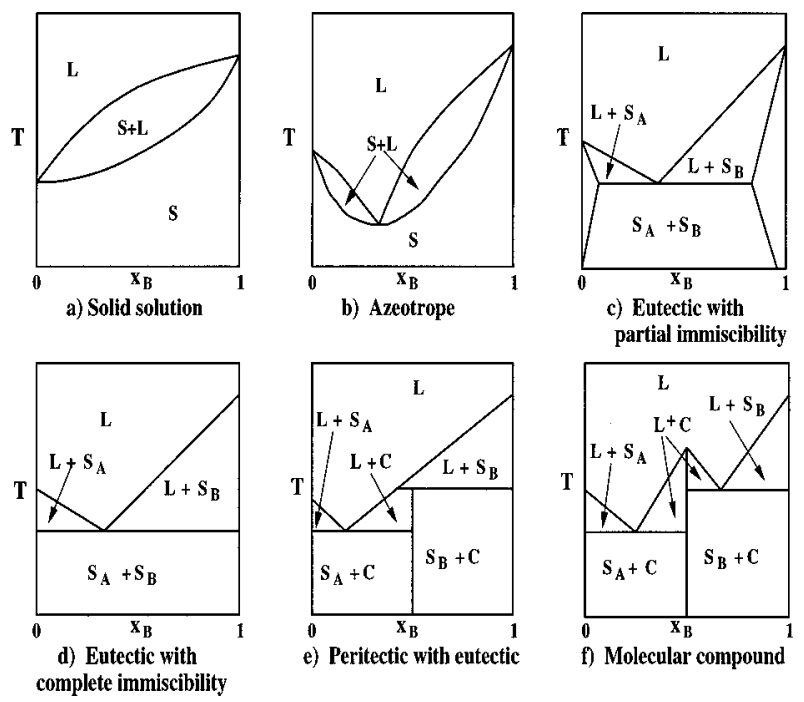

FIG. 1. The six types of solid-liquid phase diagrams identified by Matsuoka: ${ }^{1}$ (a) solid solution, (b) azeotrope, (c) eutectic with partial immiscibility, (d) eutectic with complete immiscibility, (e) peritectic with eutectic, and (f) molecular compound. The phase diagrams are shown in the $T-x_{\mathrm{B}}$ plane where $x_{\mathrm{B}}$ is the mole fraction of component $\mathrm{B}$. The symbols are: $\mathrm{L}=$ liquid mixture of $\mathrm{A}$ and $\mathrm{B}, \mathrm{S}=$ solid solution of $\mathrm{A}$ and $\mathrm{B}, \mathrm{S}_{\mathrm{A}}=$ solid solution rich in $\mathrm{A}, \mathrm{S}_{\mathrm{B}}=$ solid solution rich in $\mathrm{B}, \mathrm{C}=$ ordered solid with fixed stoichiometric ratio $\mathrm{A}_{m} \mathrm{~B}_{n}$.

Cottin and Monson also calculated phase diagrams for binary Lennard-Jones liquid mixtures ${ }^{6}$ coexisting with substitutionally disordered fcc binary Lennard-Jones solids. They calculated phase diagrams for argon-methane, argonkrypton, and krypton-methane, and compared them with experimental results. They found that the shape of the phase diagram predicted by cell theory is similar to the shape of the phase diagram observed experimentally.

Another approach to calculating solid-liquid equilibria is density functional theory. In density functional theory, a crystalline solid is treated as if it were an inhomogeneous liquid. ${ }^{7}$ There seem to be two main approaches to developing analytical expressions for the free energy of the solid: truncated perturbation expansions in which the excess free energy is expanded about a uniform reference liquid, ${ }^{8,9}$ and nonperturbative effective liquid approximations in which the excess free energy of the nonuniform liquid (crystalline solid) is approximated by the excess free energy of a uniform liquid evaluated at an effective density. ${ }^{10,11}$ The liquid solution free energy can be obtained from an equation of state. As in the cell theory, once the free energies of both phases are obtained, the convex envelope construction method is used to locate the solid-liquid phase envelope.

Density functional theory has been applied to the calculation of phase diagrams for hard-sphere and Lennard-Jones binary mixtures. Rick and Haymet, ${ }^{8,9}$ Denton and Ashcroft, ${ }^{11}$ and Zeng and Oxtoby ${ }^{10}$ used density functional theory to calculate phase diagrams for binary hard-sphere liquid mixtures coexisting with substitutionally disordered fec hardsphere solid solutions. Rick and Haymet, and Denton and Ashcroft also investigated the range of stability for several ordered binary hard-sphere solid solutions coexisting with a liquid, where the composition of each phase was fixed at $x$
$=0.5$. The nonperturbative theories (Denton and Ashcroft, Zeng and Oxtoby) give more accurate results than the perturbative theories (Rick and Haymet). As was the case with the cell theory, the density functional theory applied to binary hard-sphere mixtures shows that size differences alone can determine the shape of the phase diagram. Density functional theory is not as successful as cell theory at predicting phase behavior in binary Lennard-Jones systems. Rick and Haymet used density functional theory to calculate solidliquid phase diagrams for binary Lennard-Jones mixtures of argon-methane, argon-krypton, and krypton-methane, and compared them with experimental results. Of the three systems, they were only able to predict the shape of the krypton-methane phase diagram correctly.

It is common practice to test molecularly-based theories, such as cell theory and density functional theory, with molecular simulation data. Since molecular simulations are exact calculations for a given molecular model, they provide a direct test of theoretical approximations. The approach taken in most simulations of solid-liquid phase equilibrium involves calculating the free energy of each phase separately and then determining the coexistence curve using the convex envelope construction method. Since the free energy cannot be directly measured in a simulation, thermodynamic integration techniques are often used. In thermodynamic integration, the free energy of a phase is calculated by numerically integrating along a reversible path from a reference state to the desired state. This integration requires simulation data at many intermediate state points. The liquid phase free energy can be calculated by thermodynamic integration from the ideal gas reference state. The solid phase free energy cannot be determined by thermodynamic integration from the ideal gas state because this path crosses a first-order transition, where appreciable hysteresis may occur, and is irreversible. ${ }^{12}$ There are, however, other free energy reference states that can be used. Frenkel and Ladd ${ }^{13}$ have used the Einstein crystal as a reference state to calculate the free energy of a hardsphere solid via thermodynamic integration. An Einstein crystal consists of regularly-spaced molecules that are held in place on a lattice by the repulsive forces between their nearest neighbors. ${ }^{14}$ Eldridge et al. ${ }^{15-17}$ have used Frenkel and Ladd's Einstein crystal integration method to calculate the free energies of binary hard-sphere solids with ordered phases such as $A B, A B_{2}$, and $A B_{13}$. Frenkel and Ladd's integration method is not well-suited for substitutionally disordered solid phases, i.e., solid solutions, because there are relatively small free energy differences between the real mixture and the Einstein reference mixture. ${ }^{18}$

Other thermodynamic integration approaches have been taken for solid-liquid equilibrium calculations when the solid is a disordered crystal. Kranendonk and Frenkel ${ }^{18,19}$ developed two methods for calculating the free energies of disordered fcc binary hard-sphere solid solutions using the free energy of a pure hard sphere fcc solid, as calculated by Frenkel and Ladd, as the reference state. In one method, they construct a reversible path from the pure hard-sphere fcc solid reference by fixing the density and diameter ratio and varying the composition from unity to the composition of the hard-sphere mixture using particle insertion techniques. In a 
TABLE I. Diameter ratios for binary hard-sphere mixtures that exhibit solid-liquid phase diagrams of the solid solution, azeotrope, and eutectic types. All of the solid phases are substitutionally disordered solid solutions except for eutectics with complete immiscibility, in which case the solid phases contain only one component.

\begin{tabular}{llcc}
\hline \hline \multicolumn{1}{c}{ Phase diagram } & \multicolumn{1}{c}{ Cell theory ${ }^{\mathrm{a}}$} & $\begin{array}{c}\text { Thermodynamic } \\
\text { integration }^{\mathrm{b}}\end{array}$ & $\begin{array}{c}\text { Semigrand } \\
\text { Monte Carlo }^{\mathrm{c}}\end{array}$ \\
\hline $\begin{array}{l}\text { Solid solution } \\
\text { Solid solution/azeotrope boundary }\end{array}$ & $0.95,0.97$ & 0.95 & 0.95 \\
Azeotrope & $0.9,0.92,0.93,0.94$ & 0.9425 & $0.9,0.92$ \\
Azeotrope/eutectic boundary & & 0.875 & $0.9,0.93$ \\
Eutectic with partial immiscibility & 0.85 & 0.85 & \\
Eutectic with complete immiscibility & $0.62,0.73,0.8$ & & \\
\hline \hline
\end{tabular}

${ }^{\mathrm{a} R e f e r e n c e} 4$.

${ }^{\mathrm{b}}$ Reference 19.

${ }^{\mathrm{c}}$ Reference 20 .

second method, they construct a reversible path by fixing the density and composition and varying the diameter ratio from unity to the diameter ratio of the hard-sphere mixture. Kofke $^{20}$ introduced another thermodynamic integration method for calculating solid-liquid coexistence for cases in which the solid is a disordered binary hard-sphere fcc solid solution. This method utilizes semigrand canonical ensemble simulations $^{21,22}$ in which the number of molecules, temperature, pressure, and species fugacity fractions are fixed and the density and composition are allowed to vary. Kofke used semigrand canonical simulations to generate composition versus fugacity fraction data for several solid phase isotherms. Using this data and the chemical potential of one of the pure species as a reference (taken from the data of Hoover and Ree), ${ }^{23}$ Kofke integrated the semigrand canonical fundamental equation to calculate the species chemical potential as a function of fugacity fraction for each isotherm. The chemical potential versus fugacity fraction curve for the liquid phase was obtained from a hard-sphere equation of state. Since the species chemical potentials and fugacity fractions must be equal at equilibrium, phase coexistence could be found for each isotherm by determining the intersection of the chemical potential versus fugacity fraction curves.

Using cell theory and the molecular simulation techniques described above, several workers have determined the range of size ratios in binary hard-sphere mixtures that yield the various types of phase diagrams. Table I lists hard-sphere diameter ratios that produce solid-liquid phase diagrams of the solid solution, azeotrope, and eutectic types, along with the diameter ratio boundaries between these three types. These substitutionally disordered solids are generally stable with respect to phase separation at diameter ratios greater than 0.85 . For lower diameter ratios substitutionally ordered

TABLE II. Range of diameter ratios at which binary hard-sphere mixtures form stable substitutionally ordered solid solutions of the types $A B_{13}, A B_{2}$, and $A B$.

\begin{tabular}{ccc}
\hline \hline Solid structure & Cell theory $^{\mathrm{a}}$ & ${\text { Einstein } \text { crystal }^{\mathrm{b}}}$ \\
\hline$A B_{13}$ & $0.54-0.61$ & $0.474-0.626$ \\
$A B_{2}$ & $0.42-0.59$ & $0.4-0.62$ \\
$A B$ & $0.2-0.42$ & - \\
\hline \hline
\end{tabular}

${ }^{\mathrm{a} R e f e r e n c e} 4$.

${ }^{\mathrm{b}}$ References 16, 17. solids are stable. Table II gives the range of diameter ratios over which the structures, $A B, A B_{2}$, and $A B_{13}$ are stable in hard-sphere mixtures.

To our knowledge, the only simulation study of solidliquid equilibrium in binary Lennard-Jones mixtures is that of Vlot et $_{\text {al. }}{ }^{24}$ who calculated solid-fluid phase diagrams for symmetric (equal diameters, $\sigma_{11}=\sigma_{22}$; equal attractions, $\epsilon_{11}=\epsilon_{22}$ ) binary Lennard-Jones mixtures using a combination of molecular simulation and theory. Cross interactions between the components were defined as $\epsilon_{12}=e \sqrt{\epsilon_{11} \epsilon_{22}}$ and $\sigma_{12}=s\left(\sigma_{11}+\sigma_{22}\right) / 2$ where the parameters $e$ and $s$ represent deviations from the Lorentz-Berthelot mixing rules. ${ }^{25}$ They used a variety of thermodynamic integration and particle insertion techniques to calculate free energies. The solid phase free energy was calculated using the Einstein crystal reference state, ${ }^{26}$ the liquid phase free energy was calculated using the $f g$ sampling method, ${ }^{27,28}$ and the gas phase free energy was calculated using Widom's test particle insertion. ${ }^{29}$ The excess free energy versus composition data for all of the phases was fit with a two-parameter Redlich-Kister polynomial. ${ }^{30}$ The convex envelope construction method was used to determine the temperature versus composition phase diagrams.

In the simulation techniques that we have discussed thus far, phase equilibrium is calculated indirectly in that many simulations must be conducted at uninteresting state points in order to get one coexistence point. Prior to 1987, direct simulation of phase equilibrium was considered to be too computationally expensive because the presence of an interface requires the system to contain an unusually large number of particles. Then in 1987, Panagiotopoulos ${ }^{31}$ introduced the Gibbs ensemble method for direct simulation of phase coexistence in single component fluids and in 1988, extended the method to phase coexistence in multicomponent mixtures. ${ }^{32}$ In this method the coexisting phases are simulated independently, so as to remove the interface, yet are essentially coupled together in order to satisfy the criteria of phase equilibrium: equal temperatures, pressures, and species chemical potentials. These conditions are satisfied by simulating each phase at the same temperature and pressure, and performing particle exchanges between the phases to maintain chemical potential equality. The Gibbs ensemble method has been widely used to study vapor-liquid and liquid-liquid phase equilibria. However, because a large 
number of successful transfers of particles between each phase are required for chemical potential equilibration, ${ }^{33}$ the Gibbs ensemble method is not an efficient method for studying solid-liquid phase equilibrium.

Inspired by the Gibbs ensemble method, Kofke $e^{34,35}$ introduced the Gibbs-Duhem integration technique for direct simulation of phase equilibria. As in the Gibbs ensemble method, two or more coexisting phases are simulated independently at the same temperature and pressure. However, instead of using particle exchanges, Kofke maintains chemical potential equality in each phase by integrating along the Clapeyron equation for coexistence during the simulations. Eliminating the need for particle transfers between phases makes the Gibbs-Duhem integration method well-suited for calculating phase equilibrium for cases in which one of the phases is a solid. The Gibbs-Duhem integration simulation approach has been used to tackle a number of multicomponent, multiphase equilibrium problems, including: finding the triple point of the Lennard-Jones fluid, ${ }^{36,37}$ determining the melting line of $\mathrm{C}_{60},{ }^{38}$ and calculating phase diagrams for colloids in polymer solution ${ }^{39}$ and for polymer-induced protein precipitation. ${ }^{40}$ In each case the appropriate Clapeyron differential equation is derived and an initial coexistence condition is obtained from theory, experimental data, or a Gibbs ensemble Monte Carlo simulation.

In this paper, we combine Kofke's Gibbs-Duhem integration technique with semigrand canonical Monte Carlo simulations to calculate solid-liquid phase diagrams for binary Lennard-Jones mixtures. To our knowledge, these are the first direct simulations of solid-liquid equilibrium in binary Lennard-Jones mixtures.

We calculate solid-liquid phase diagrams for binary Lennard-Jones mixtures with diameter ratios ranging from 0.85 to 1 and attractive well-depth ratios ranging from 0.45 to 1.6 , at a reduced pressure equivalent to $1 \mathrm{~atm}$. We determine the cross-species interaction parameters by using the Lorentz-Berthelot ${ }^{25}$ mixing rules. The solid-liquid equilibrium line is calculated for each mixture by numerically integrating the Clapeyron differential equation for binary mixture phase equilibria at constant pressure. The initial condition for the integration is that of a single component Lennard-Jones liquid in equilibrium with a single component Lennard-Jones fcc crystalline solid at atmospheric pressure. The coexistence properties at subsequent integration points are determined by semigrand canonical Monte Carlo simulations (constant temperature, pressure, total number of molecules, and fugacity fraction) of the liquid and the fcc solid phases.

Highlights of our simulation results are the following. In a test of the cell theory predictions for model argonmethane, argon-krypton, and krypton-methane systems, we found that cell theory can qualitatively predict the shape of phase diagrams for Lennard-Jones mixtures. Comparison of our simulation results for the argon-krypton Lennard-Jones system with hard-sphere results at the same diameter ratio indicated that the presence of attractive interactions can change the type of phase diagram observed from azeotrope (hard-sphere) to solid solution (Lennard-Jones). This suggested that attractive interactions are an important factor in determining the type of solid-liquid phase behavior observed and prompted a more thorough investigation of the effect of variations in well-depth ratio on solid-liquid phase diagrams. We simulated 56 binary Lennard-Jones mixtures over the range of parameters listed above to determine how solid-liquid phase diagrams change as a function of diameter ratio and well-depth ratio. We found that for well-depth ratios of unity (equal attractions among species) phase behavior indicative of azeotropes and eutectics is observed for diameter ratios ranging from 0.85 to 1 . We then varied the well-depth ratio of the mixtures at several constant diameter ratios and observed transitions from azeotrope to solid solution, from azeotrope to eutectic, and from solid solution to simple peritectic. Using our simulation results, we are able to map out the boundaries separating regimes of solid solution, azeotrope, and eutectic solid-liquid phase behavior in the space spanned by the Lennard-Jones diameter ratio and welldepth ratios.

The remainder of the paper is organized as follows. Section II reviews the Gibbs-Duhem integration method and gives details of how we applied the technique to our study of binary solid-liquid mixture equilibrium. In Sec. III, the simulation results are presented and discussed. A brief summary and further discussion are given in Sec. IV.

\section{METHOD}

In this section we describe how we calculated solidliquid equilibria in binary Lennard-Jones mixtures using the Gibbs-Duhem integration method. We begin by presenting a brief overview of the Gibbs-Duhem integration method. We then discuss our procedures for determining an initial coexistence condition and integrating the Clapeyron equation. Finally, we describe the details of the semigrand ensemble simulations used throughout the integration procedure.

In the Gibbs-Duhem integration method, ${ }^{34,35,41}$ phase coexistence is determined by numerically integrating the Clapeyron differential equation appropriate to the system of interest. Clapeyron equations describe how field variables (variables that must be equal among coexisting phases) change along the phase equilibrium line. The Clapeyron equation for equilibrium between a binary solid mixture and a binary liquid mixture at constant pressure is ${ }^{35}$

$$
\frac{d \beta}{d \xi_{2}}=\frac{\left(x_{2}^{l}-x_{2}^{s}\right)}{\xi_{2}\left(1-\xi_{2}\right)\left(h^{l}-h^{s}\right)},
$$

where $\beta$ is the reciprocal temperature, $1 / k T$, with $k$ the Boltzmann constant and $T$ the absolute temperature, $\xi_{2}$ is the fugacity fraction of species $2, \xi_{2} \equiv \hat{f}_{2} / \Sigma \hat{f}_{i}$, with $\hat{f}_{i}$, the fugacity of species $i$ in solution, $x_{2}$ is the mole fraction of species $2, h$ is the molar enthalpy, and the superscripts $l$ and $s$ denote the liquid and solid phases, respectively. The righthand side of Eq. (1) can be integrated numerically to find an equation for $\beta$ as a function of $\xi_{2}$ if we have an initial condition describing the temperature, fugacity fraction, enthalpies, and compositions at one coexistence point. 


\section{A. Initial condition}

We need an initial coexistence condition before we can begin a Gibbs-Duhem integration calculation of solid-liquid equilibrium in a binary mixture. A good choice is the solidliquid equilibrium condition for either of the pure components. ${ }^{35}$ Since the slope of the integrand in Eq. (1) is undefined for pure components $\left(\xi_{2}=0, x_{2}=0\right.$ and $\xi_{2}=1$, $x_{2}=1$ ), we instead estimate it using the limiting case of infinite dilution. Here we follow Mehta and Kofke, ${ }^{42}$ who used the infinite dilution case to start their Gibbs-Duhem integration calculations of vapor-liquid equilibria in binary mixtures.

Consider the case of pure species 1 solid-liquid coexistence. We can estimate $\left(d \beta / d \xi_{2}\right)_{x_{2}=0}$ by supposing that the real mixture displays ideal solution behavior at the limit of infinite dilution of species 2 . With this assumption, the abundant component (species 1) in the ideal solution follows the Lewis Randall rule

$$
\hat{f}_{1}=x_{1} f_{1},
$$

while the dilute component (species 2) obeys Henry's law

$$
\hat{f}_{2}=x_{2} H_{2},
$$

where $\hat{f}_{1}$ and $\hat{f}_{2}$ are fugacities of species 1 and 2 in solution, $f_{1}$ is the fugacity of pure component 1 at the temperature and pressure of the mixture, and $H_{2}$ is the Henry's law constant for species 2. Letting $x_{1} \rightarrow 1$ and $x_{2} \rightarrow 0$, the fugacity fraction of species 2 becomes, $\xi_{2}=x_{2} H_{2} / f_{1}$. After making these substitutions into Eq. (1) we get

$$
\left.\frac{d \beta}{d \xi_{2}}\right|_{x_{2}=0}=\frac{\hat{f}_{2} / H_{2}^{l}-\hat{f}_{2} / H_{2}^{s}}{\left(x_{2} H_{2} / f_{1}\right)\left(1-x_{2} H_{2} / f_{1}\right)\left(h^{l}-h^{s}\right)},
$$

which, after simplification, leads to

$$
\left.\frac{d \beta}{d \xi_{2}}\right|_{x_{2}=0}=\frac{f_{1}\left(1 / H_{2}^{l}-1 / H_{2}^{s}\right)}{\left(h^{l}-h^{s}\right)} .
$$

This gives us an estimate of the integrand at the initial condition of $\xi_{2}=0$ and melting temperature, $T_{1}$, of pure species 1 .

We can calculate all of the quantities on the right-hand side of Eq. (5) with an NPT (constant number particles, N, pressure, $\mathrm{P}$, and temperature, $\mathrm{T}$ ) simulation of pure species 1 . The molar enthalpy of each phase is $h=\langle u+P v\rangle_{\mathrm{NPT}}$, where $u$ is the configurational energy of the system and the brackets, \langle\rangle$_{\mathrm{NPT}}$, denote an NPT ensemble average. The quantity $f_{1} / H_{2}$ is given by ${ }^{42}$

$$
\frac{f_{1}}{H_{2}}=\left\langle\exp \left(-\beta \Delta u_{1 \rightarrow 2}\right)\right\rangle_{\mathrm{NPT}},
$$

where $\Delta u_{1 \rightarrow 2}$ is the exchange energy associated with switching a particle from species 1 to 2 . The exchange energy, $\Delta u_{1 \rightarrow 2}$, can be obtained by conducting trial identity switches during the simulation. A trial identity switch consists of randomly selecting a particle and calculating the en- ergy that would result if we were to switch the particle from species 1 to species 2 . This is done without actually changing the particle's identity.

\section{B. Integration}

Once we have an initial coexistence condition, we can begin the Gibbs-Duhem integration series using a predictor-corrector algorithm to integrate Eq. (1) over the entire range of fugacity fractions, $\xi_{2}=0$ to $\xi_{2}=1$. Stepping to the next fugacity fraction, $\left(\xi_{2}\right)_{1}$, from the initial data point, the next reciprocal temperature, $\beta_{1}$, can be estimated using the trapezoid-rule predictor formula

$$
\beta_{1}^{(0)}=\beta_{0}+\left[\left(\xi_{2}\right)_{1}-\left(\xi_{2}\right)_{0}\right] F\left(\beta_{0},\left(\xi_{2}\right)_{0}\right),
$$

where the " 0 ", superscript indicates that $\beta_{1}^{(0)}$ (a predicted value) is our zeroth iteration attempt at finding the reciprocal temperature $\beta_{1},\left(\xi_{2}\right)_{0}$ and $\beta_{0}$ are the initial fugacity fraction and reciprocal temperature, respectively, and $F$ is the righthand side of Eq. (1) evaluated at the initial condition. Once $\beta_{1}^{(0)}$ is estimated at the given fugacity fraction $\left(\xi_{2}\right)_{1}$, two semigrand canonical (NPT $\xi_{2}$ ) Monte Carlo simulations (one for the liquid phase and one for the solid phase) are conducted in order to equilibrate the configurations and to calculate the enthalpies and mole fractions at the new state point. (Details of the NPT $\xi_{2}$ simulations will be given in Sec. II C.)

After each phase is equilibrated we refine our estimate for $\beta_{1}$ with the trapezoid-rule corrector

$$
\begin{aligned}
\beta_{1}^{(1)}= & \beta_{0}+\frac{\left[\left(\xi_{2}\right)_{1}-\left(\xi_{2}\right)_{0}\right]}{2}\left[F_{1}^{(0)}\left(\beta_{1}^{(0)},\left(\xi_{2}\right)_{1}\right)\right. \\
& \left.+F_{0}\left(\beta_{0},\left(\xi_{2}\right)_{0}\right)\right]
\end{aligned}
$$

where the subscripts " 0 " and " 1 " denote the initial and current conditions, respectively, and $F_{1}^{(0)}$ is calculated from simulation averages of the enthalpies and mole fractions at $\beta_{1}^{(0)}$ and $\left(\xi_{2}\right)_{1}$. After the reciprocal temperature is corrected, two NPT $\xi_{2}$ simulations, one for each phase, are started simultaneously and running averages of the compositions and enthalpies are calculated. Each simulation is paused periodically and the running averages from the simulations to that point are used to calculate $F_{1}^{(i)}$ and correct the reciprocal temperature to obtain the $(i+1)$ th estimate, $\beta_{1}^{(i+1)}$. This pattern continues until the temperature estimate stops varying within a specified tolerance. A final production segment of simulations are run at the temperature determined from the corrector segment to obtain the final average enthalpies and mole fractions for the coexistence point.

After $\beta_{1}$ converges and the production runs are completed, the fugacity fraction is incremented to the next state point and the predictor-corrector algorithm described above is repeated. Higher order predictor-corrector equations are used as we obtain more coexistence points. The midpoint predictor-corrector ${ }^{35}$ is used once two state points are known, 


$$
\begin{aligned}
& \beta_{n+1}^{(0)}=\beta_{n-1}+2\left[\left(\xi_{2}\right)_{n+1}-\left(\xi_{2}\right)_{n}\right] F_{n}\left(\beta_{n},\left(\xi_{2}\right)_{n}\right), \\
& \beta_{n+1}^{(i+1)}=\beta_{n-1}+\frac{\left[\left(\xi_{2}\right)_{n+1}-\left(\xi_{2}\right)_{n}\right]}{3}\left[F_{n+1}^{(i)}+4 F_{n}+F_{n-1}\right],
\end{aligned}
$$

and the modified Adams predictor-corrector ${ }^{43}$ is used once three or more state points are known,

$$
\begin{aligned}
\beta_{n+1}^{(0)}= & \beta_{n}+\frac{\left[\left(\xi_{2}\right)_{n+1}-\left(\xi_{2}\right)_{n}\right]}{24}\left[55 F_{n}-59 F_{n-1}\right. \\
& \left.+37 F_{n-2}-9 F_{n-3}\right], \\
\beta_{n+1}^{(i+1)}= & \beta_{n}+\frac{\left[\left(\xi_{2}\right)_{n+1}-\left(\xi_{2}\right)_{n}\right]}{24}\left[9 F_{n+1}^{(i)}+19 F_{n}\right. \\
& \left.-5 F_{n-1}+F_{n-2}\right] .
\end{aligned}
$$

In these sets of equations the predictor is listed first. The subscripts denote the coexistence conditions determined from simulations with $(n+1)$ being the current simulation and the superscripts denote the iterations of the corrector for the current coexistence point. We mapped the entire temperature versus composition phase diagram by repeating the predictor-corrector algorithm from $\xi_{2}=0$ to $\xi_{2}=1$.

\section{Simulations}

The enthalpies and mole fractions needed as input to the integration of Eq. (1) are obtained from semigrand canonical (constant $\mathrm{NPT} \xi_{2}$ ) Monte Carlo computer simulations. ${ }^{22}$ In this work, all simulations were run with a system size of 500 particles at a reduced pressure equivalent to $1 \mathrm{~atm}$. The temperature and fugacity fraction were varied according to the values specified by the Gibbs-Duhem integration predictorcorrector. There are three types of Monte Carlo trial moves in semigrand canonical simulations: particle displacements, volume change moves, and particle identity exchanges. The particle displacements and volume change moves are conducted just as they are in a standard NPT simulation. ${ }^{44}$ In the particle identity exchange moves, a particle is selected at random and given a trial species identity switch. The identity switch is accepted according to the ratio of the species fugacity fractions, $\xi_{1}$ and $\xi_{2}$. The overall acceptance probability ${ }^{42}$ for the moves in the $\mathrm{NPT} \xi_{2}$ ensemble is $\min [1, \exp (\Lambda)]$ where

$$
\begin{aligned}
\Lambda= & -\beta\left(U^{\text {trial }}-U^{\text {old }}\right)-\beta P\left(V^{\text {trial }}-V^{\text {old }}\right) \\
& +N \ln \frac{V^{\text {trial }}}{\mathrm{V}^{\text {old }}}+m \ln \frac{\xi_{2}}{1-\xi_{2}} .
\end{aligned}
$$

In Eq. (13), $U^{\text {trial }}$ and $U^{\text {old }}$, and $V^{\text {trial }}$ and $V^{\text {old }}$ are the configurational energies and volumes of the trial and existing states, respectively, $m=+1$ if the trial identity switch is from species 1 to 2 , and $m=-1$ if the trial identity switch is from species 2 to 1 . In NPT $\xi_{2}$ simulations the choice of the type of Monte Carlo move is made randomly but weighted such that the ratio of attempted moves is one volume change to $N$ particle displacements to $N$ identity switches. The length of the simulation is given in cycles, where one cycle represents either $N$ displacement attempts, $N$ identity switch attempts, or one volume change attempt. In our work, a typi-
TABLE III. Lennard-Jones potential parameters for argon, krypton, and methane.

\begin{tabular}{lcc}
\hline \hline & $\epsilon_{i i} / k(\mathrm{~K})$ & $\sigma_{i i}(A)$ \\
\hline $\mathrm{Ar}$ & 141.2 & 3.336 \\
$\mathrm{Kr}$ & 191.4 & 3.575 \\
$\mathrm{CH} 4$ & 159.7 & 3.706 \\
\hline \hline
\end{tabular}

cal NPT $\xi_{2}$ simulation is equilibrated for 15000 cycles and then followed by a production run of 3000 cycles to compute the average enthalpy and mole fraction. The only difference between solid and liquid phase simulations is that to maintain an fcc crystalline structure in the solid phase simulations we impose a single occupancy constraint ${ }^{20,45}$ on the trial displacements of particles in the solid, i.e., any displacements that put the particle outside its lattice cell are rejected.

Other details of the NPT $\xi_{2}$ simulations are as follows. The simulation volume is a cubic box with periodic boundary conditions. The particles interact via the Lennard-Jones potential model

$$
u_{i j}(r)=4 \epsilon_{i j}\left[\left(\frac{\sigma_{i j}}{r}\right)^{12}-\left(\frac{\sigma_{i j}}{r}\right)^{6}\right],
$$

where $u_{i j}$ is the potential energy of interaction between particles $i$ and $j, r$ is the distance between particles $i$ and $j, \epsilon_{i j}$ is the Lennard-Jones attractive well-depth, and $\sigma_{i j}$ is the Lennard-Jones diameter. We determine the crossspecies interaction parameters $\left(\sigma_{12}, \epsilon_{12}\right)$ by using the Lorentz-Berthelot ${ }^{25}$ mixing rules $\sigma_{12}=\left(\sigma_{11}+\sigma_{22}\right) / 2$ and $\epsilon_{12}=\sqrt{\epsilon_{11} \epsilon_{22}}$. The potential interactions are truncated at a cutoff radius of half the box length. To compensate for this truncation, a long range correction is applied to the energy and virial calculations during the simulation by assuming a uniform density distribution beyond the cutoff radius. ${ }^{44}$

\section{RESULTS AND DISCUSSION}

In this section we describe the results of our GibbsDuhem integration calculation of solid-liquid phase diagrams for binary Lennard-Jones systems. We begin by comparing our simulation results with cell theory results and with experimental data. Since our molecular simulations are exact calculations for the Lennard-Jones potential model we can compare the simulation data to experimental results to test the ability of the Lennard-Jones model to mimic behavior in real systems. We can also compare the simulation data to the cell theory results to test the cell theory's ability to predict the behavior of the Lennard-Jones system. We then examine how variations in the ratios of Lennard-Jones diameters $\left(\sigma_{11} / \sigma_{22}\right)$ and well-depths $\left(\epsilon_{11} / \epsilon_{22}\right)$ influence the types of phase diagrams that will be observed.

\section{A. Comparison with molecular theory and experiment}

Following Cottin and Monson, ${ }^{6}$ we calculated temperature-composition diagrams for the model mixtures: argonmethane, argon-krypton, and krypton-methane, at $P$ $=1 \mathrm{~atm}$. These phase diagrams were then compared with Cottin and Monson's cell theory predictions and with experimental data. 


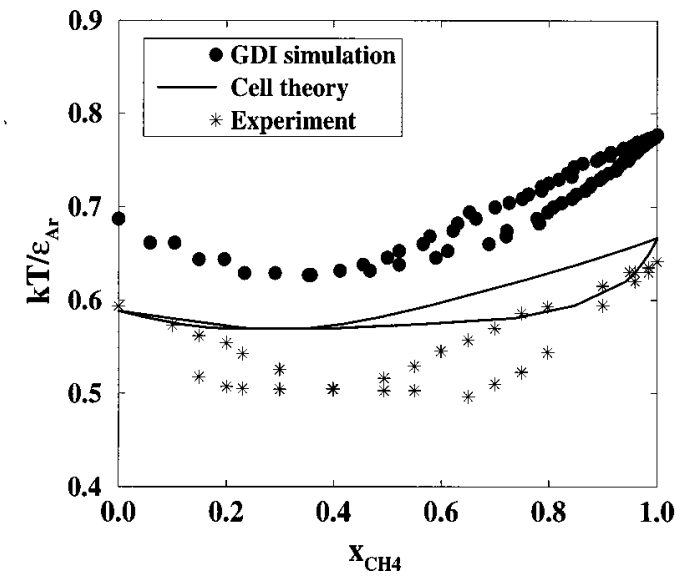

FIG. 2. Temperature vs. composition solid-liquid phase diagram for the argon-methane system at $1 \mathrm{~atm}$. The filled circles represent Gibbs-Duhem integration (GDI) simulations, the solid line corresponds to the Cottin and Monson's cell theory ${ }^{6}$ prediction, and the stars correspond to van't Zelfde et $a l .{ }^{46}$ experiments.

We used the same Lennard-Jones parameters as Cottin and Monson; the diameter $\left(\sigma_{i i}\right)$ and well-depth $\left(\epsilon_{i i}\right)$ for argon, krypton, and methane are listed in Table III. We worked in reduced units $T^{*}=k T / \epsilon_{11}$ and $P^{*}=P \sigma_{11}^{3} / \epsilon_{11}$. For $P$ $=1 \mathrm{~atm}$, the corresponding reduced pressures are $P^{*}$ $=0.00193$ (argon), $P^{*}=0.00175$ (krypton), and $P^{*}$ $=0.00243$ (methane).

The initial condition for the Gibbs-Duhem integration calculations came from the simulation data of Agrawal and Kofke $^{37}$ who calculated the solid-liquid equilibrium line for a single component Lennard-Jones system. They obtained a reduced melting temperature of $T^{*}=0.687$ for the range of reduced pressures, $P^{*}=0.0008$ to 0.008 . In our calculations, we started with an initial condition of $T_{1}^{*}=k T_{1} / \epsilon_{11}$ $=0.687$, where $T_{1}^{*}$ and $T_{1}$ are the reduced and absolute melting temperatures of component 1 , respectively. We estimated the uncertainty in the simulation data by performing three Gibbs-Duhem integration simulations for each mixture and calculating the standard deviation of the temperature and mole fractions of the liquid and solid phases at each coexistence point. In all three mixtures, the standard deviation in coexistence temperature, $k T / \epsilon_{11}$, is less than $0.2 \%$ and in mole fraction, $x_{2}$, is less than $0.6 \%$.

Figure 2 shows the temperature-composition phase diagram for the argon-methane system obtained using GibbsDuhem integration starting from pure methane solid-liquid coexistence, along with the cell theory prediction of Cottin and Monson and the experimental results of van't Zelfde et al. $;^{46}$ the cell theory and experimental data have been reduced with the Lennard-Jones parameters given in Table III. The diameter ratio is $\sigma_{\mathrm{Ar}} / \sigma_{\mathrm{CH}_{4}}=0.9$ and the well-depth ratio is $\epsilon_{\mathrm{Ar}} / \epsilon_{\mathrm{CH}_{4}}=0.88$. We obtain a minimum azeotrope similar to the experimental data and cell theory prediction. A glance at the comparison of the ability of simulations and theory to predict experimental behavior would, at first, lead one to think that the theory is more accurate than the simulations. However, as Cottin ${ }^{4}$ points out, direct comparison of theoretical predictions with experimental data is difficult because inadequacies in the potential model coupled with simplifying

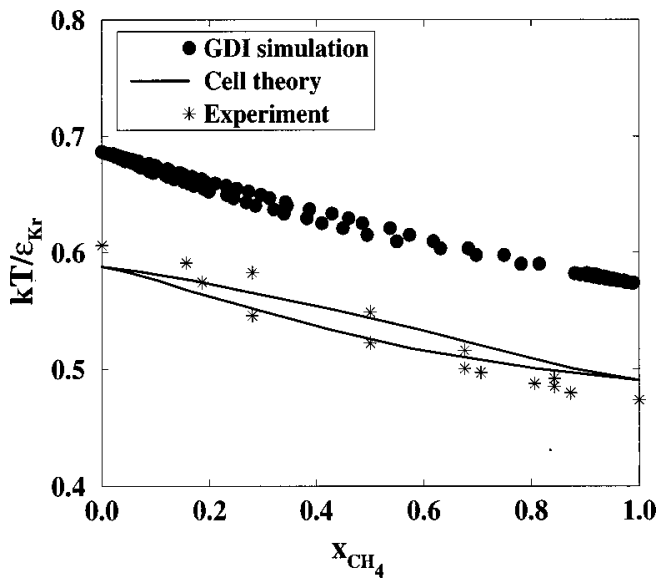

FIG. 3. Temperature vs. composition solid-liquid phase diagram for the krypton-methane system at $1 \mathrm{~atm}$. The filled circles represent GibbsDuhem integration (GDI) simulations, the solid line corresponds to the Cottin and Monson's cell theory ${ }^{6}$ prediction, and the stars correspond to Veith and Schroder's ${ }^{47}$ experiments.

approximations made in the theory could lead to error cancellation. This cancellation effect is illustrated in Fig. 2 where the Lennard-Jones model (simulation) over predicts melting temperatures for real systems (experiments), and cell theory under predicts melting temperatures for the LennardJones model. There is a good agreement in the predicted azeotrope composition between cell theory and the LennardJones model, although neither predicts the azeotrope composition found in experiments. After making these comparisons, it is also useful to compare the phase diagram of this Lennard-Jones mixture with a hard-sphere mixture of the same diameter ratio to determine whether attractive interactions are contributing to the type of phase behavior observed. In this case, a hard-sphere mixture with the diameter ratio $\sigma_{\mathrm{Ar}} / \sigma_{\mathrm{CH}_{4}}=0.9$ would also display azeotrope phase behavior.

Figure 3 shows the temperature-composition phase diagram of the krypton-methane system obtained using GibbsDuhem integration starting from pure methane solid-liquid coexistence, along with the cell theory prediction and the experimental results of Veith and Schroder. ${ }^{47}$ The diameter ratio is $\sigma_{\mathrm{Kr}} / \sigma_{\mathrm{CH}_{4}}=0.96$ and the well-depth ratio is $\epsilon_{\mathrm{Kr}} / \epsilon_{\mathrm{CH}_{4}}$ $=1.2$. We obtain a spindle-shaped phase diagram very similar to the experimental data and cell theory prediction. A hard-sphere mixture with the diameter ratio $\sigma_{\mathrm{Kr}} / \sigma_{\mathrm{CH}_{4}}=0.96$ would also display solid solution phase behavior.

Figure 4 shows the temperature-composition phase diagram for the argon-krypton system obtained using GibbsDuhem integration starting from pure argon solid-liquid coexistence, along with the cell theory prediction and the experimental results of Heastie. ${ }^{48}$ Here the diameter ratio is $\sigma_{\mathrm{Ar}} / \sigma_{\mathrm{Kr}}=0.93$ and the well-depth ratio is $\epsilon_{\mathrm{Ar}} / \epsilon_{\mathrm{Kr}}=0.74$. We obtain a spindle-shaped phase diagram similar to the experimental data and the cell theory prediction. The simulation matches the shape of the experimental data, especially in the argon-rich region where the temperature-composition line tends to level off. A hard-sphere mixture with a diameter ratio $\sigma_{\mathrm{Ar}} / \sigma_{\mathrm{Kr}}=0.93$ would not exhibit solid solution behavior but instead would have a minimum azeotrope. This suggests 


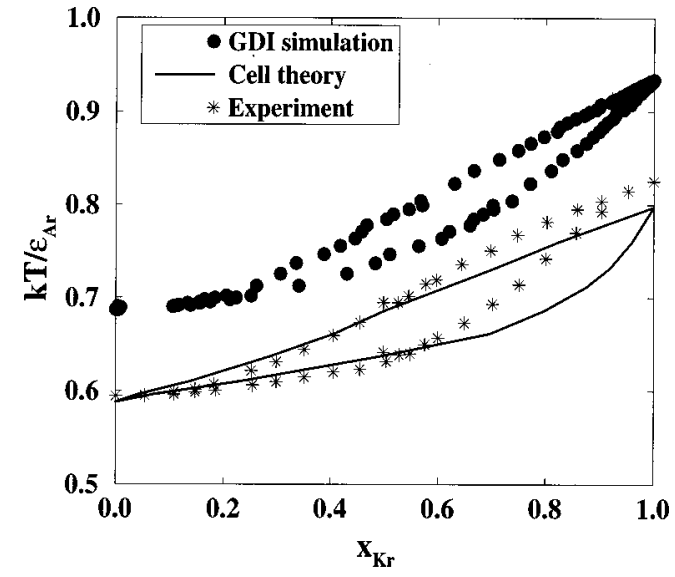

FIG. 4. Temperature vs. composition solid-liquid phase diagram for the argon-krypton system at $1 \mathrm{~atm}$. The filled circles represent Gibbs-Duhem integration (GDI) simulations, the solid line corresponds to the Cottin and Monson's cell theory ${ }^{6}$ prediction, and the stars correspond to Heastie's ${ }^{48}$ experiments.

that the intermolecular attractions between molecules can be a significant factor in determining the type of solid-liquid phase diagram observed.

\section{B. Influence of attractions on phase behavior}

As we have discussed in Sec. I, five of the six different types of solid-liquid phase diagrams for binary mixtures can be predicted by using a hard-sphere model with an appropriate diameter ratio. Of the three Lennard-Jones mixtures discussed thus far, we have seen one instance (argon-krypton) where the difference in attractive interactions was large enough to change the type of phase diagram observed from azeotrope (hard sphere) to solid solution (Lennard-Jones). In order to get a better idea of what role attractive interactions play in solid-liquid mixture phase equilibria, we performed a systematic investigation of how solid-liquid phase diagrams change as a function of both Lennard-Jones diameter ratio $\left(\sigma_{11} / \sigma_{22}\right)$ and well-depth ratio $\left(\epsilon_{11} / \epsilon_{22}\right)$.

All of the phase diagrams presented in this section were calculated at a reduced pressure, $P^{*}=0.002$, which corresponds to a real pressure of 1 atm using the Lennard-Jones parameters for argon. Due to the large number of mixtures investigated, we did not estimate error bars for the simulation data but we expect the uncertainty to be similar to the uncertainty estimated for the simulation data in Figs. 2-4.

To begin our investigation, we examined two limiting cases: sizes are equal $\left(\sigma_{11} / \sigma_{22}=1\right)$ while attractions are varied, and attractions are equal $\left(\epsilon_{11} / \epsilon_{22}=1\right)$ while sizes are varied. The ultimate limiting case, a binary mixture with $\sigma_{11} / \sigma_{22}=1$ and $\epsilon_{11} / \epsilon_{22}=1$, is uninteresting; the temperature-composition phase diagram would simply be a horizontal line at $T^{*}=0.687$ since the components are indistinguishable. Phase diagrams were calculated for binary mixtures with a diameter ratio $\sigma_{11} / \sigma_{22}=1$ and well-depth ratios $\epsilon_{11} / \epsilon_{22}=0.65,0.75,0.85,0.90$, and 0.95 . Figure 5 shows temperature-composition phase diagrams for these binary mixtures. All of the mixtures form solid solutions with a spindle shape. As $\epsilon_{11} / \epsilon_{22}$ decreases (attractions among spe-

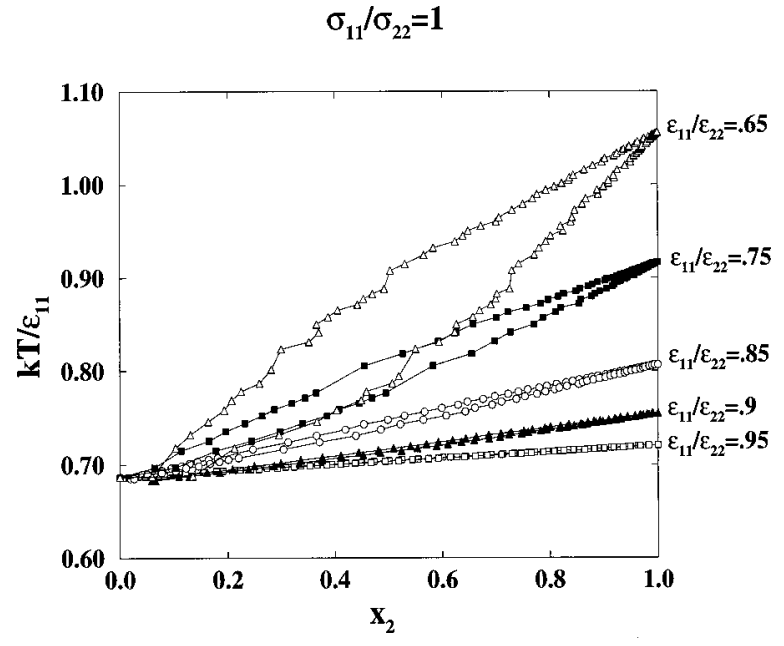

FIG. 5. Temperature vs. composition solid-liquid phase diagrams for Lennard-Jones binary mixtures at diameter ratio, $\sigma_{11} / \sigma_{22}=1.0$ and welldepth ratios $\epsilon_{11} / \epsilon_{22}=0.65,0.75,0.85,0.90$, and 0.95 . Lines are drawn through the simulation points for clarity.

cies 2 particles become stronger), the melting point temperature of species 2 increases and the degree of phase separation (width of the spindle) increases.

We also calculated phase diagrams for binary mixtures with a well-depth ratio $\epsilon_{11} / \epsilon_{22}=1$ and diameter ratios $\sigma_{11} / \sigma_{22}=0.85,0.86,0.87,0.88,0.9,0.91,0.93,0.94$, and 0.95 . Figure 6 shows selected temperature-composition phase diagrams for these binary mixtures. An azeotrope phase diagram is obtained for $\sigma_{11} / \sigma_{22}=0.95$. As $\sigma_{11} / \sigma_{22}$ decreases from 0.95 to 0.86 , the azeotrope temperature decreases and the degree of phase separation on either side of the azeotrope composition increases. The azeotrope temperature decreases as $\sigma_{11} / \sigma_{22}$ decreases because for increasing size differences, the liquid phase becomes more stable than the solid phase. ${ }^{49}$ At $\sigma_{11} / \sigma_{22}=0.86$, the solidus line takes a steep drop in temperature over the composition range $x_{2}$ $=0$ to 0.05 , and as a result, the shape of the azeotrope appears distorted. At $\sigma_{11} / \sigma_{22}=0.85$ the solidus line is no

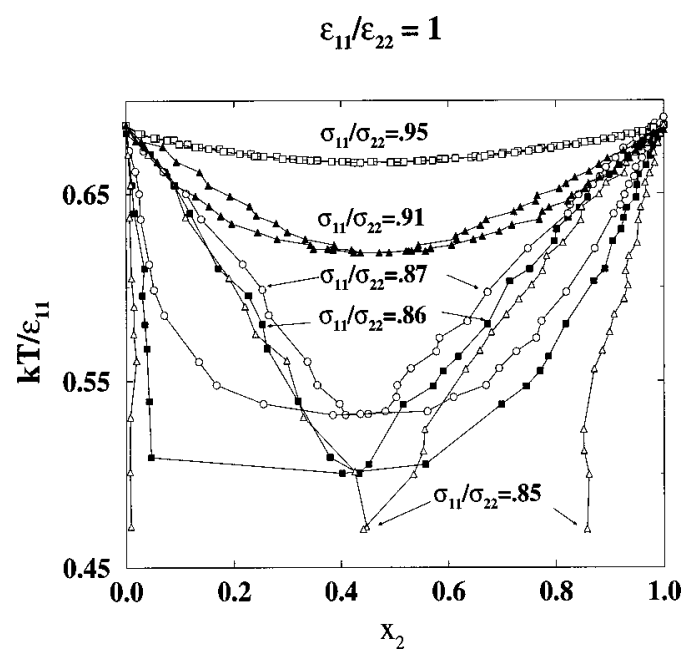

FIG. 6. Temperature vs. composition solid-liquid phase diagrams for Lennard-Jones binary mixtures at well-depth ratio $\epsilon_{11} / \epsilon_{22}=1.0$ and diameter ratios, $\sigma_{11} / \sigma_{22}=0.85,0.86,0.87,0.91$, and 0.95 . 


$$
\sigma_{11} / \sigma_{22}=\mathbf{0 . 9 2 5}
$$

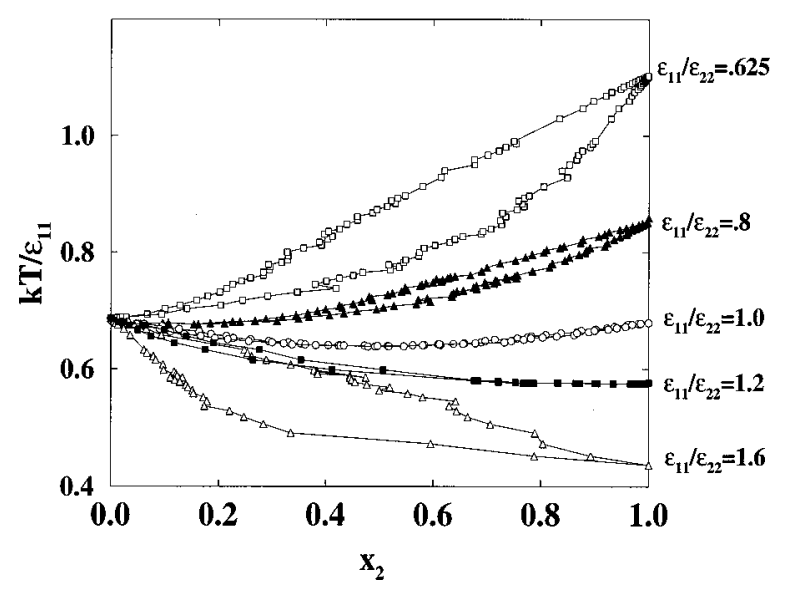

FIG. 7. Temperature vs. composition solid-liquid phase diagrams for Lennard-Jones binary mixtures at diameter ratio, $\sigma_{11} / \sigma_{22}=0.925$ and welldepth ratios $\epsilon_{11} / \epsilon_{22}=0.625,0.8,1.0,1.2$, and 1.6.

longer continuous across the entire range of composition and a eutectic phase diagram is obtained. Thus, we conclude that an azeotrope to eutectic transition occurs between $\sigma_{11} / \sigma_{22}$ $=0.85$ and 0.86. In comparison, Kranendonk and Frenkel ${ }^{19}$ found an azeotrope to eutectic transition for hard-sphere mixtures at $\sigma_{11} / \sigma_{22}=0.875$.

Next, we attempted to establish boundaries for solid solution, azeotrope, and eutectic phase behavior in $\epsilon_{11} / \epsilon_{22}$ versus $\sigma_{11} / \sigma_{22}$ parameter space. We calculated five series of phase diagrams at diameter ratios $\sigma_{11} / \sigma_{22}=0.85,0.875,0.9$, 0.925 , and 0.95 . Within each $\sigma_{11} / \sigma_{22}$ series, the well-depth ratio $\epsilon_{11} / \epsilon_{22}$ was varied from 0.45 to 1.6 .

In the first series (not shown), we calculated phase diagrams for binary mixtures with $\sigma_{11} / \sigma_{22}=0.95$ and $\epsilon_{11} / \epsilon_{22}$ $=0.55,0.65,0.75,1.0,1.1,1.2,1.3,1.4$, and 1.6. We found solid solution phase diagrams for all the mixtures in this series, except at $\epsilon_{11} / \epsilon_{22}=1.0$ where, as described above, the

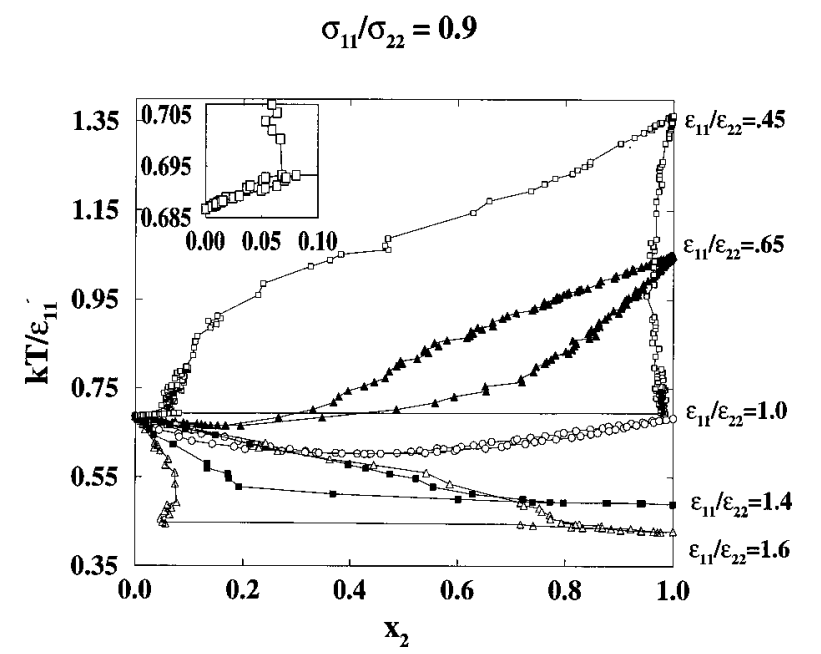

FIG. 8. Temperature vs. composition solid-liquid phase diagrams for Lennard-Jones binary mixtures at diameter ratio, $\sigma_{11} / \sigma_{22}=0.9$ and welldepth ratios $\epsilon_{11} / \epsilon_{22}=0.45,0.65,1.0,1.4$, and 1.6. The inset shows the region around the peritectic temperature at $x_{2}=0-0.1$ for $\epsilon_{11} / \epsilon_{22}=0.45$.

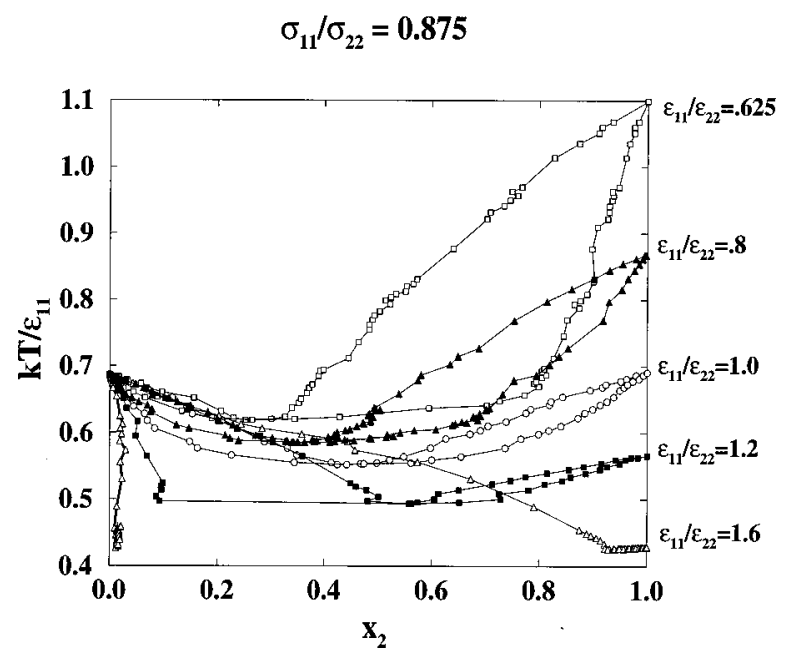

FIG. 9. Temperature vs. composition solid-liquid phase diagrams for Lennard-Jones binary mixtures at diameter ratio, $\sigma_{11} / \sigma_{22}=0.875$ and welldepth ratios $\epsilon_{11} / \epsilon_{22}=0.625,0.8,1.0,1.2$, and 1.6.

phase diagram displayed a minimum azeotrope. As $\epsilon_{11} / \epsilon_{22}$ moves away from unity, the width of the spindle increases. A hard-sphere mixture with this diameter ratio has a solid solution phase diagram.

In the second series, we calculated phase diagrams for binary mixtures with $\sigma_{11} / \sigma_{22}=0.925$ and $\epsilon_{11} / \epsilon_{22}=0.625$, $0.8,1.0,1.2,1.4$, and 1.6. Figure 7 shows selected temperature-composition phase diagrams for binary mixtures from this series. An azeotrope phase diagram is obtained for $\epsilon_{11} / \epsilon_{22}=1.0$. As $\epsilon_{11} / \epsilon_{22}$ decreases from 1 to 0.8 , the melting point of pure component 2 increases and the azeotrope temperature increases. When the azeotrope temperature is equal to the melting point temperature of pure component 1 $\left(\epsilon_{11} / \epsilon_{22}=0.625\right)$, the azeotrope disappears and the phase diagram becomes a solid solution. Conversely, as $\epsilon_{11} / \epsilon_{22}$ increases from 1 to 1.2 , the melting point temperature of pure component 2 decreases and the azeotrope temperature decreases. When the azeotrope is equal to the melting point of pure component $2\left(\epsilon_{11} / \epsilon_{22}>1.2\right)$, the azeotrope disappears and the phase diagram becomes a solid solution. As $\epsilon_{11} / \epsilon_{22}$ increases from 1.2 to 1.6 , the melting point of species 2 decreases and the degree of phase separation increases. A hard-sphere mixture with this diameter ratio has an azeotrope phase diagram.

In the third series, we calculated phase diagrams for binary mixtures with $\sigma_{11} / \sigma_{22}=0.9$ and $\epsilon_{11} / \epsilon_{22}=0.45,0.65$, $0.85,0.9,1.0,1.05,1.1,1.2,1.4$, and 1.6. Figure 8 shows selected temperature-composition phase diagrams for binary mixtures from this series. An azeotrope phase diagram is obtained for $\epsilon_{11} / \epsilon_{22}=1.0$. As $\epsilon_{11} / \epsilon_{22}$ decreases from 1 to 0.65 , the melting point of pure component 2 increases and the azeotrope temperature increases. As $\epsilon_{11} / \epsilon_{22}$ decreases from 0.65 to 0.45 , the azeotrope disappears and the phase diagram becomes a simple peritectic. A simple peritectic phase diagram is characterized by a peritectic temperature at which the liquid composition is either greater than or less than the compositions of the two solid phases with which it coexists. The inset in Fig. 8 shows the region of the peritectic 


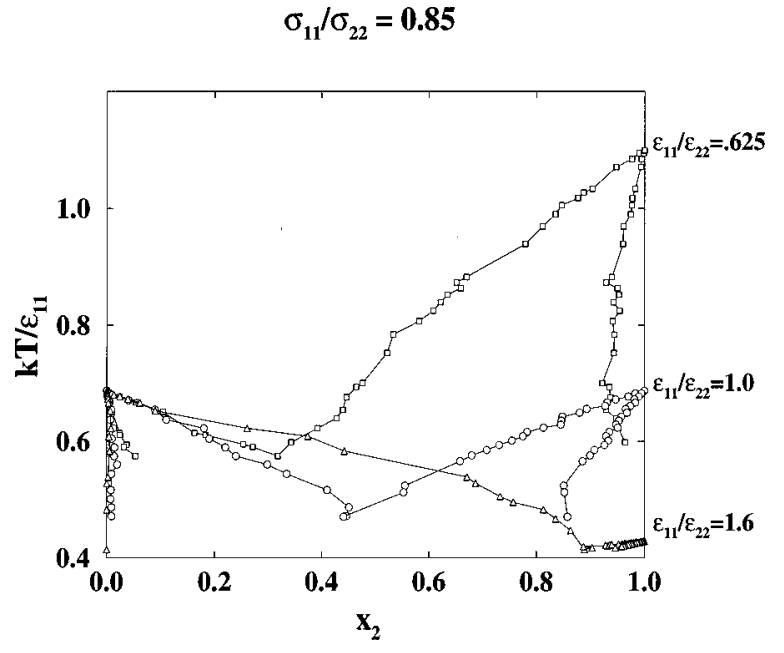

FIG. 10. Temperature vs. composition solid-liquid phase diagrams for Lennard-Jones binary mixtures at diameter ratio, $\sigma_{11} / \sigma_{22}=0.85$ and welldepth ratios $\epsilon_{11} / \epsilon_{22}=0.625,1.0$, and 1.6.

phase diagram between the compositions, $x_{2}=0-0.1$. As $\epsilon_{11} / \epsilon_{22}$ increases from 1 to 1.4 , the melting point temperature of pure component 2 decreases and the azeotrope temperature decreases. When the azeotrope temperature is equal to the melting point of pure component 2, the azeotrope disappears and the phase diagram becomes a solid solution. As $\epsilon_{11} / \epsilon_{22}$ increases from 1.4 to 1.6, the phase diagram changes from a solid solution to a simple peritectic. A hard-sphere mixture with this diameter ratio has an azeotrope phase diagram.

In the fourth series, we calculated phase diagrams for binary mixtures with $\sigma_{11} / \sigma_{22}=0.875$ and $\epsilon_{11} / \epsilon_{22}=0.625$, $0.8,1,1.2,1.4$, and 1.6. Figure 9 shows selected temperature-composition phase diagrams for binary mixtures from this series. An azeotrope phase diagram is obtained for $\epsilon_{11} / \epsilon_{22}=1.0$. As $\epsilon_{11} / \epsilon_{22}$ decreases from 1 to 0.625 , the melting point of pure component 2 increases and the azeotrope temperature increases. As $\epsilon_{11} / \epsilon_{22}$ increases from 1 to 1.2 , the melting point temperature of pure component 2 decreases and the azeotrope temperature decreases. At $\epsilon_{11} / \epsilon_{22}$ $=1.2$, the solidus line takes a steep drop in temperature over the composition range $x_{2}=0$ to 0.1 and as a result, the shape of the azeotrope appears distorted. As $\epsilon_{11} / \epsilon_{22}$ increases from 1.2 to 1.6 , the phase diagram changes from an azeotrope to a eutectic. A hard-sphere mixture with this diameter ratio shows a transition between azeotrope and eutectic phase behavior.

In the fifth and final series, we calculated phase diagrams for binary mixtures with $\sigma_{11} / \sigma_{22}=0.85$ and $\epsilon_{11} / \epsilon_{22}$ $=0.625,0.8,0.9,1.0,1.1$, and 1.6. Figure 10 shows selected temperature-composition phase diagrams for binary mixtures from this series. A eutectic phase diagram is obtained for $\epsilon_{11} / \epsilon_{22}=1.0$. As $\epsilon_{11} / \epsilon_{22}$ decreases from 1 to 0.625 , the melting point of pure component 2 increases and the eutectic temperature increases. As $\epsilon_{11} / \epsilon_{22}$ increases from 1 to 1.6, the melting point temperature of pure component 2 decreases and the eutectic temperature decreases. A hard-sphere mixture with this diameter ratio has a eutectic phase diagram.

In Fig. 11, we show the regions of $\sigma_{11} / \sigma_{22}$ vs. $\epsilon_{11} / \epsilon_{22}$

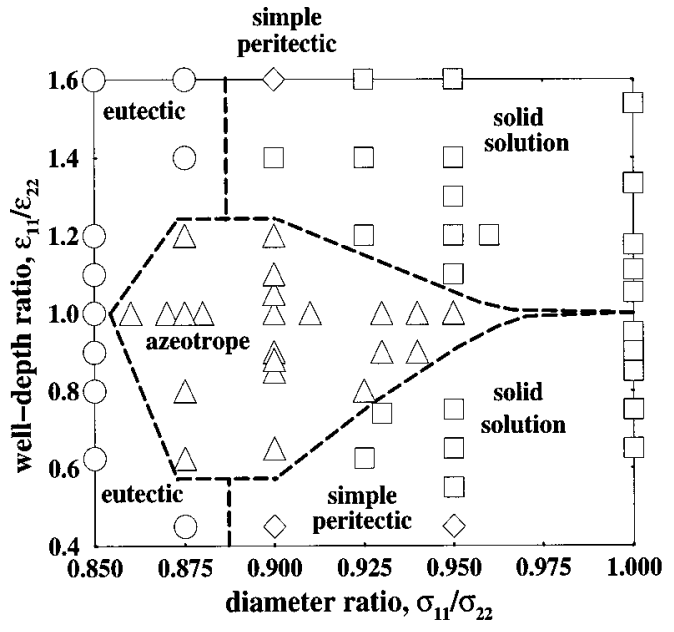

FIG. 11. Boundaries (dashed lines) separating regions of solid solutions (squares), azeotropes (triangles), eutectics (circles), and simple peritectics (diamonds) in Lennard-Jones parameter space, $0.45 \leqslant \epsilon_{11} / \epsilon_{22} \leqslant 1.6$ and $0.85 \leqslant \sigma_{11} / \sigma_{22} \leqslant 1.0$.

parameter space where the four types of solid-liquid phase diagrams (solid solutions, azeotropes, eutectics, and simple peritectics) are found for the Lennard-Jones model. Each mixture studied in this paper is given a symbol corresponding to the type of phase diagram calculated via a GibbsDuhem integration simulation. We did not calculate phase diagrams for $\sigma_{11} / \sigma_{22}=1$ and $\epsilon_{11} / \epsilon_{22}>1$ because due to symmetry the phase diagrams in this region will be identical to the phase diagrams for $\epsilon_{11} / \epsilon_{22}<1$.

Using these simulation results, boundaries separating the regions of solid solution, azeotrope, and eutectic phase behavior have been estimated. Boundaries for the peritectic region have not been estimated because simple peritectics occurred only a few times. Between $\sigma_{11} / \sigma_{22}=0.95$ and 1 , solid solution type phase diagrams are found everywhere except for a narrow region near $\epsilon_{11} / \epsilon_{22}=1$ where azeotropes are formed because the melting points of the pure components are nearly equal. ${ }^{25}$ As $\sigma_{11} / \sigma_{22}$ decreases below 0.95 , this azeotropic region broadens and then narrows again as $\sigma_{11} / \sigma_{22}$ approaches 0.86 , forming a fish-shaped region whose maximum height spans the range $\epsilon_{11} / \epsilon_{22}=0.6$ to 1.2. The region outside of the azeotrope region switches from solid solution to eutectic as the diameter ratio $\sigma_{11} / \sigma_{22}$ decreases below 0.9 .

To understand how and why variations in the attractive interactions lead to the boundaries shown in the $\epsilon_{11} / \epsilon_{22}$ versus $\sigma_{11} / \sigma_{22}$ parameter space displayed in Fig. 11, it is helpful to consider the schematic phase diagrams for the selected mixtures shown in Fig. 12. The columns correspond to mixtures with constant diameter ratios, $\sigma_{11} / \sigma_{22}=0.875,0.9$, and 0.925 , and the rows correspond to mixtures with constant well-depth ratios, $\epsilon_{11} / \epsilon_{22}=0.625,1.0$, and 1.6. The solidliquid lines are based on our simulation results. The solidsolid equilibrium lines are based on our best guess as to how the upper critical solution temperature of the solid-solid immiscibility dome shifts with variations in well-depth ratio. Underpinning this guess is quasichemical theory, ${ }^{50,51}$ which tells us that the upper critical solution temperature increases 

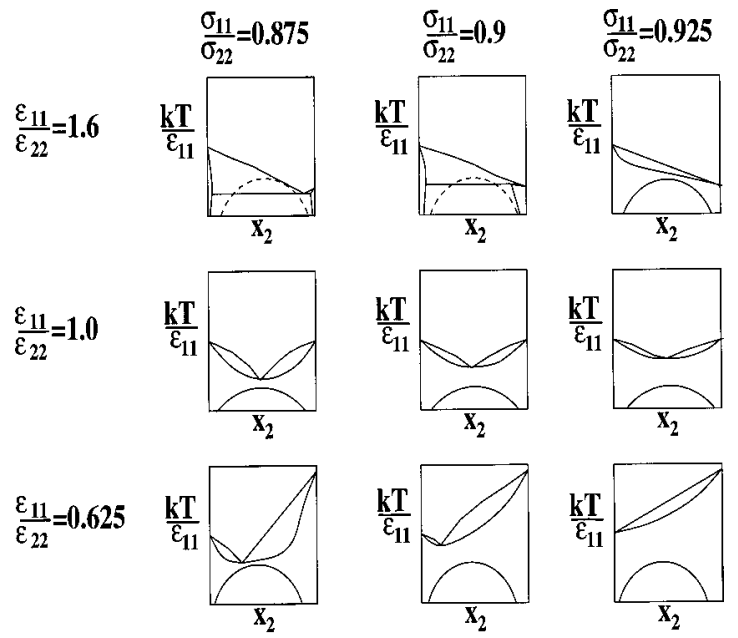

FIG. 12. Schematic phase diagrams showing how variations in diameter ratio and well-depth ratio lead changes in the phase diagram. The columns correspond to mixtures with constant diameter ratios, $\sigma_{11} / \sigma_{22}=0.875,0.9$, and 0.925 , and the rows correspond to mixtures with constant well-depth ratios, $\epsilon_{11} / \epsilon_{22}=0.625,1.0$, and 1.6. The solid-liquid lines were calculated in this work. The solid-solid lines have not been calculated for these mixtures, but we have guessed how the upper critical solution temperature shifts with variation in well-depth ratio using quasichemical theory.

as the attractions between like molecules become stronger than the attractions between unlike molecules. This means that the upper critical solution temperature will increase as $\epsilon_{11} / \epsilon_{22}$ increases above unity or decreases below unity. This is shown schematically in Fig. 12.

In the $\sigma_{11} / \sigma_{22}=0.925$ column, the phase diagram is a solid solution at $\epsilon_{11} / \epsilon_{22}=1.6$. As the well-depth ratio $\epsilon_{11} / \epsilon_{22}$ decreases, the melting point temperature of component 2 increases and the solid-liquid lines "pull away" from the solid-solid immiscibility dome. In the $\sigma_{11} / \sigma_{22}=0.9$ column, the phase diagram is a simple peritectic at $\epsilon_{11} / \epsilon_{22}$ $=1.6$. As the well-depth ratio decreases, the solid-liquid lines move away from the underlying solid-solid immiscibility dome (shown in dashed lines) and the phase diagram becomes an azeotrope. In the $\sigma_{11} / \sigma_{22}=0.875$ column, the phase diagram is a eutectic at $\epsilon_{11} / \epsilon_{22}=1.6$. As the welldepth ratio decreases, the solid-liquid lines move away from the solid-solid immiscibility dome and the phase diagram becomes an azeotrope.

In the $\epsilon_{11} / \epsilon_{22}=1.6$ row, the phase diagram is a solid solution at $\sigma_{11} / \sigma_{22}=0.925$. As the diameter ratio decreases, the solid-liquid lines "fall" because the liquid phase can accommodate the larger differences in size easier than the solid phase ${ }^{49}$ and the phase diagram becomes a simple peritectic at $\sigma_{11} / \sigma_{22}=0.9$ and a eutectic at $\sigma_{11} / \sigma_{22}=0.875$. In the $\epsilon_{11} / \epsilon_{22}=1.0$ row, the phase diagram is an azeotrope at $\sigma_{11} / \sigma_{22}=0.925$. As the diameter ratio decreases and the solid-liquid lines fall, the azeotrope temperature decreases. In the $\epsilon_{11} / \epsilon_{22}=0.625$ row, the phase diagram is a solid solution at $\sigma_{11} / \sigma_{22}=0.925$. As the diameter ratio decreases and the solid-liquid lines fall, the phase diagram becomes an azeotrope.

\section{SUMMARY}

The Gibbs-Duhem integration technique was combined with semigrand canonical Monte Carlo simulations to calculate solid-liquid phase diagrams for binary Lennard-Jones mixtures.

We calculated phase diagrams for model mixtures: argon-methane, argon-krypton, and krypton-methane, and compared them to cell theory predictions and experimental data. We found that cell theory qualitatively predicts the shape of phase diagrams for Lennard-Jones mixtures. Comparison of our simulation results for the argon-krypton Lennard-Jones system with hard-sphere results at the same diameter ratio $\left(\sigma_{\mathrm{Ar}} / \sigma_{\mathrm{Kr}}=0.93\right)$ indicated that the presence of attractive interactions can change the type of phase diagram observed from azeotrope (hard-sphere) to solid solution (Lennard-Jones). This suggested that we further explore the effect of asymmetry in the attractions on the types of phase diagrams observed.

We simulated 56 binary Lennard-Jones mixtures over a range of diameter ratios $\sigma_{11} / \sigma_{22}=0.85-1.0$ and well-depth ratios $\epsilon_{11} / \epsilon_{22}=0.45-1.6$. We found that for well-depth ratios of unity (equal attractions among species), phase behavior indicative of azeotropes and eutectics is observed for diameter ratios ranging from 0.85 to 1 . Much to our surprise, we also found that varying the attractive interactions at a fixed diameter ratio can perturb the type of solid-liquid phase diagram obtained, a trend that previously ${ }^{6,8}$ had not been explored in Lennard-Jones mixtures.

There are several areas for further study. First, it would be helpful to know where the solid-solid immiscible dome is located relative to the solid-liquid lines that we have calculated in this paper. Determining solid-solid equilibrium lines would give a more complete picture of how the solid-liquid and solid-solid equilibrium lines merge as we vary parameters and observe transitions between solid solutions, azeotropes, eutectics, and simple peritectics. Second, it would be interesting to repeat this type of investigation for $\sigma_{11} / \sigma_{22}$ $<0.85$, a region where several ordered solid phases are known to be stable.

${ }^{1}$ M. Matsuoka, Bunri Gijutsu 7, 245 (1977).

${ }^{2}$ X. Cottin and P. A. Monson, J. Chem. Phys. 99, 8914 (1993).

${ }^{3}$ X. Cottin and P. A. Monson, J. Chem. Phys. 102, 3354 (1995).

${ }^{4}$ X. Cottin, Ph.D. thesis, University of Massachusetts, 1996.

${ }^{5}$ X. Cottin, E. P. A. Paras, C. Vega, and P. A. Monson, Fluid Phase Equilibria 117, 114 (1996).

${ }^{6}$ X. Cottin and P. A. Monson, J. Chem. Phys. 105, 10022 (1996).

${ }^{7}$ D. W. Oxtoby, Nature (London) 347, 725 (1990).

${ }^{8}$ S. W. Rick and A. D. J. Haymet, J. Chem. Phys. 90, 1188 (1989).

${ }^{9}$ S. W. Rick and A. D. J. Haymet, J. Phys. Chem. 94, 5212 (1990)

${ }^{10}$ X. C. Zeng and D. W. Oxtoby, J. Chem. Phys. 93, 4357 (1990).

${ }^{11}$ A. R. Denton and N. W. Ashcroft, Phys. Rev. A 42, 7312 (1990).

${ }^{12}$ D. Frenkel and B. Smit, Understanding Molecular Simulations (Academic, San Diego, 1996).

${ }^{13}$ D. Frenkel and A. J. C. Ladd, J. Chem. Phys. 81, 3188 (1984).

${ }^{14}$ R. L. Rowley, Statistical Mechanics for Thermophysical Property Calculations (Prentice Hall, Engelwood Cliffs, New Jersey, 1994).

${ }^{15}$ M. D. Eldridge, P. A. Madden, and D. Frenkel, Nature (London) 365, 35 (1993).

${ }^{16}$ M. D. Eldridge, P. A. Madden, and D. Frenkel, Mol. Phys. 79, 105 (1993).

${ }^{17}$ M. D. Eldridge, P. A. Madden, and D. Frenkel, Mol. Phys. 80, 987 (1993).

${ }^{18}$ W. G. T. Kranendonk and D. Frenkel, Mol. Phys. 72, 699 (1991).

${ }^{19}$ W. G. T. Kranendonk and D. Frenkel, Mol. Phys. 72, 679 (1991). 
${ }^{20}$ D. A. Kofke, Mol. Simul. 7, 285 (1991).

${ }^{21}$ D. A. Kofke and E. D. Glandt, J. Chem. Phys. 87, 4881 (1987).

${ }^{22}$ D. A. Kofke and E. D. Glandt, Mol. Phys. 64, 1105 (1988).

${ }^{23}$ W. G. Hoover and F. H. Ree, J. Chem. Phys. 49, 3609 (1968).

${ }^{24}$ M. J. Vlot, J. C. van Miltenburg, H. A. J. Oonk, and J. P. van der Eerden, J. Chem. Phys. 107, 10102 (1997).

${ }^{25}$ J. S. Rowlinson, Liquids and Liquid Mixtures (Butterworth Scientific, London, 1982).

${ }^{26}$ M. J. Vlot and J. P. van der Eerden, J. Chem. Phys. 106, 2771 (1997).

${ }^{27}$ K. S. Shing and K. E. Gubbins, Mol. Phys. 46, 1109 (1982).

${ }^{28}$ M. J. Vlot, S. Claassen, H. E. A. Huitema, and J. P. van der Eerden, Mol. Phys. 91, 19 (1997)

${ }^{29}$ B. Widom, J. Chem. Phys. 39, 2808 (1963).

${ }^{30}$ J. M. Prausnitz, R. N. Lichtenthaler, and E. G. de Azevedo, Molecular Thermodynamics of Fluid-Phase Equilibria, 2nd ed. (Prentice Hall, Englewood Cliffs, New Jersey, 1986)

${ }^{31}$ A. Z. Panagiotopoulos, Mol. Phys. 61, 813 (1987).

${ }^{32}$ A. Z. Panagiotopoulos, N. Quirke, M. Stapleton, and D. J. Tildesley, Mol. Phys. 63, 527 (1988).

${ }^{33}$ A. Z. Panagiotopoulos, Mol. Simul. 9, 1 (1992).

${ }^{34}$ D. A. Kofke, Mol. Phys. 78, 1331 (1993).

${ }^{35}$ D. A. Kofke, J. Chem. Phys. 98, 4149 (1993).

${ }^{36}$ R. Agrawal, M. Mehta, and D. A. Kofke, Int. J. Thermophys. 15, 1073 (1994).
${ }^{37}$ R. Agrawal and D. A. Kofke, Mol. Phys. 85, 43 (1995).

${ }^{38}$ M. H. J. Hagen E. J. Meijer, G. C. A. M. Mooij, D. Frenkel, and H. N. W. Lekkerkerker, Nature (London) 365, 425 (1993).

${ }^{39}$ E. J. Meijer and D. Frenkel, J. Chem. Phys. 100, 6873 (1994).

${ }^{40}$ F. W. Tavares and S. I. Sandler, AIChE. J. 43, 218 (1997).

${ }^{41}$ D. A. Kofke, in Monte Carlo Methods in Chemistry, edited by D. M. Ferguson, J. I. Siepmann, and D. G. Truhlar (Interscience, New York, 1998), Vol. 105, Chap. 13, p. 405.

${ }^{42}$ M. Mehta and D. A. Kofke, Chem. Eng. Sci. 49, 2633 (1994).

${ }^{43}$ B. Carnahan, H. A. Luther, and J. O. Wilkes, Applied Numerical Methods (Wiley, New York, 1969).

${ }^{44}$ M. P. Allen and D. J. Tildesley, Computer Simulation of Liquids (Clarendon, Oxford, 1987).

${ }^{45}$ J.-P. Hansen and L. Verlet, Phys. Rev. 184, 151 (1969).

${ }^{46}$ P. van't Zelfde, M. H. Omar, H. G. M. le Pair-Schroten, and Z. Dokoupil, Physica (Amsterdam) 38, 241 (1968).

${ }^{47}$ H. Veith and E. Schroder, Z. Phys. Chem. Abt. A 179, 16 (1937).

${ }^{48}$ R. Heastie, Nature (London) 176, 747 (1955).

${ }^{49}$ W. Hume-Rothery, R. E. Smallman, and C. W. Haworth, The Structure of Metals and Alloys (The Metals and Metallurgy Trust, London, 1969).

${ }^{50}$ A. Prince, Alloy Phase Equilibria (Elsevier, Amsterdam, 1966).

${ }^{51} \mathrm{P}$. Gordon, Principles of Phase Diagrams in Materials Systems (Krieger, Malabar, Florida, 1983). 